\title{
Scaling of the energy spectra of turbulent channels
}

\author{
By JUAN C. DEL ÁLAMO', JAVIER JIMÉNEZ ${ }^{1,2}$, \\ PAULO ZANDONADE ${ }^{3}$ AND ROBERT D. MOSER \\ ${ }^{1}$ School of Aeronautics, Universidad Politécnica de Madrid, 28040 Madrid, Spain \\ ${ }^{2}$ Centre for Turbulence Research, Stanford University, Stanford, CA 94305, USA \\ ${ }^{3}$ Department of Theoretical and Applied Mechanics, University of Illinois, Urbana, IL 61801, USA
}

(Received 26 June 2003, and in revised form 27 October 2003)

The spectra and correlations of the velocity fluctuations in turbulent channels, especially above the buffer layer, are analysed using new direct numerical simulations with friction Reynolds numbers up to $R e_{\tau}=1900$. It is found, and explained, that their scaling is anomalous in several respects, including a square-root behaviour of their width with respect to their length, and a velocity scaling of the largest modes with the centreline velocity $U_{c}$. It is shown that this implies a logarithmic correction to the $k^{-1}$ energy spectrum, and that it leads to a scaling of the total fluctuation intensities away from the wall which agrees well with the mixed scaling of de Graaff \& Eaton (2000) at intermediate Reynolds numbers, but which tends to a pure scaling with $U_{c}$ at very large ones.

\section{Introduction}

While self-similarity is always a welcome feature in physical problems, simplifying their solution and providing insight into their behaviour, its failure in situations in which in principle it ought to apply is perhaps even more interesting, because it forces us to explain what went wrong. Some of the best-known similarity arguments in fluid dynamics are those regarding the overlap layer in wall turbulence, and it has lately become increasingly clear that, while the logarithmic law for the mean velocity profile may be a good approximation to the experimental data (Zagarola \& Smits 1997; Österlund et al. 2000), there are serious similarity failures in the behaviour of the velocity fluctuations, including the scaling of their intensity in wall units and their predicted $k^{-1}$ energy spectrum.

The reason why the intensity of the streamwise velocity fluctuations does not scale well with the friction velocity was given by Townsend (1976), who noted that wallparallel motions are inactive from the point of view of the Reynolds stresses, and do not have to scale with them. The discovery by de Graaff \& Eaton (2000) that they scale well in mixed units was however unexpected, and presents a clear theoretical challenge, because it implies that they should be describable by a well-defined model.

Even more interesting is the failure of the $k^{-1}$ energy spectrum, which can be easily predicted from an assumed lack of length scale for fluctuations whose wavelengths are short with respect to the flow thickness, but long with respect to the wall distance (Townsend 1976). It has been known since Laufer (1954) that the $k^{-1}$ law is approximately correct, but recent detailed observations by Hites (1997) and by Morrison et al. (2002) reveal important corrections which are inconsistent with the original argument. 


\begin{tabular}{lccccccccccc}
\hline & Case & $R e_{\tau}$ & $L_{x} / h$ & $L_{z} / h$ & $T U_{b} / L_{x}$ & $\Delta x^{+}$ & $\Delta z^{+}$ & $\Delta y_{c}^{+}$ & $N_{x}$ & $N_{z}$ & $N_{y}$ \\
Series 1 & $\mathrm{L} 550$ & 547 & $8 \pi$ & $4 \pi$ & 10 & 8.9 & 4.5 & 6.7 & 1536 & 1536 & 257 \\
& $\mathrm{~L} 950$ & 934 & $8 \pi$ & $3 \pi$ & 9.2 & 7.6 & 3.8 & 7.6 & 3072 & 2304 & 385 \\
Series 2 & $\mathrm{S} 550$ & 550 & $\pi$ & $\pi / 2$ & 77 & 8.9 & 4.5 & 6.7 & 192 & 192 & 257 \\
& $\mathrm{~S} 950$ & 964 & $\pi$ & $\pi / 2$ & 27 & 7.8 & 3.9 & 7.8 & 384 & 384 & 385 \\
& $\mathrm{~S} 1900$ & 1901 & $\pi$ & $\pi / 2$ & 22 & 7.8 & 3.9 & 7.8 & 768 & 768 & 769
\end{tabular}

TABLE 1. Parameters of the simulations. $T$ is the time during which the statistics were collected after discarding initial transients. $U_{b}$ is the bulk velocity. $\Delta x$ and $\Delta z$ are the collocation resolutions parallel to the wall, using $N_{x}$ and $N_{z}$ points. $\Delta y_{c}$ is the wall-normal grid spacing at the centre of the channel, and $N_{y}$ is the number of Chebychev polynomials.

The present paper looks at the reasons for those two failures, which we will find to be related. We use data from new direct numerical simulations of plane turbulent channels at moderate Reynolds numbers $R e_{\tau}=u_{\tau} h / v \leqslant 1900$, where $u_{\tau}$ is the wallfriction velocity and $h$ is the channel half-width. The new data show that the original failure of self-similarity occurs in the relation between the lengths and widths of the structures in the overlap region, and that this failure results in logarithmic corrections to the $k^{-1}$ energy spectrum. These are then demonstrated using experimental data, for which $h$ will represent the pipe radius or the $99 \%$ boundary layer thickness.

We then show, and motivate, that the velocity scale of the largest structures is not the friction velocity, but the mean velocity at the centreline, and use the resulting spectral model to derive a modified mixed scaling law for the fluctuations.

The new simulations are described in $\S 2$. The results, and their relations to the different scaling arguments, are detailed in $\S 3$, followed by a short concluding section. The companion paper by Jiménez, del Álamo \& Flores (2003) extends the present analysis to the buffer and viscous layers, and that by del Álamo et al. (2004) contains a more detailed analysis of the structures away from the wall.

\section{The numerical experiments}

The numerical code integrates the Navier-Stokes equations in the form of evolution problems for the wall-normal vorticity $\omega_{y}$ and for the Laplacian of the wall-normal velocity $\nabla^{2} v$, as in Kim, Moin \& Moser (1987). The spatial discretization uses dealiased Fourier expansions in the wall-parallel planes, and Chebychev polynomials in $y$. The streamwise and spanwise coordinates and velocity components are respectively $x, z$ and $u, w$. The temporal discretization is third-order semi-implicit Runge-Kutta, as in Moser, Kim \& Mansour (1999).

The present simulations, summarized in table 1 , are divided into two series. The runs in the first one use numerical boxes with very long periodicities $L_{x}$ and $L_{z}$, to try to account for all the energetic structures in the flow, including those in the outer region whose size is proportional to $h$, and that could not be captured accurately in previous simulations (Jiménez 1998; Kim \& Adrian 1999; del Álamo \& Jiménez 2001, 2003).

The second series of experiments focuses on the overlap layer, increasing the Reynolds number at the expense of the size of the numerical box, especially for the case S1900. Less emphasis will be put on the other two simulations in this series, which were performed mainly to assess the effect of small numerical boxes on the computed statistics. That effect cannot necessarily be neglected, since the fractions 

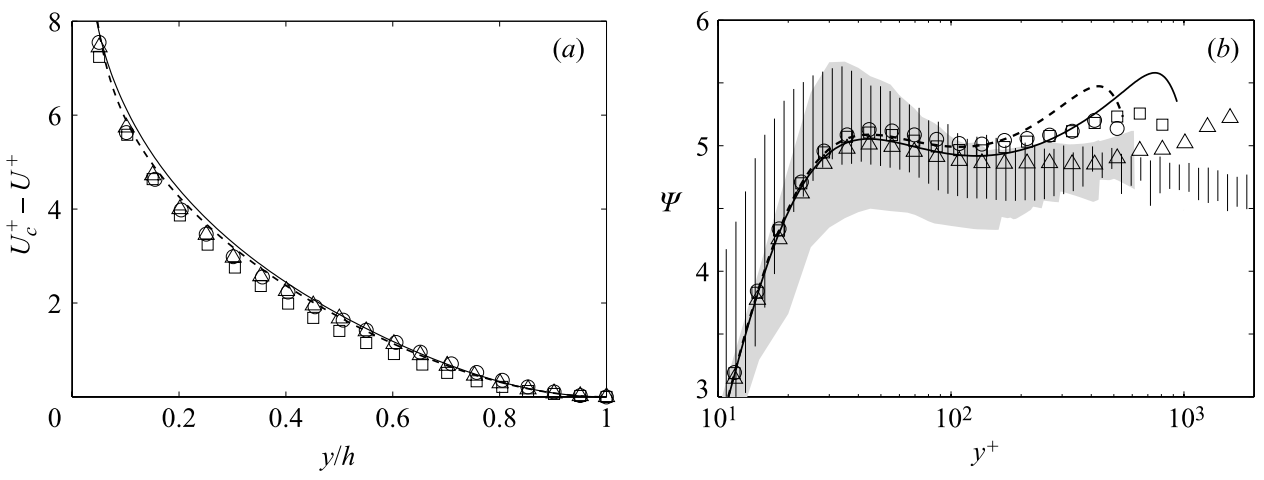

Figure 1. (a) Mean velocity defect as a function of wall distance. (b) Deviation $\Psi$ of the mean velocity from the logarithmic function. -..--, L550; ——, L950; O, S550; $\square$, S950; $\triangle$, S1900. The shaded region in $(b)$ covers the maximum scatter of experimental boundary layers from Smith (1994), $R e_{\theta}=4600-13000$, Österlund et al. (2000), $R e_{\theta}=2500-27000$, and de Graaff \& Eaton (2000), $R e_{\theta}=1430-31000$. The hatched area covers experimental pipes from Perry et al. (1986), $R e_{\tau}=1600-3800$, Durst et al. (1995), $R e_{\tau}=271-570$ and Zagarola \& Smits (1997), $R e_{\tau}=1700-5.3 \times 10^{5}$. Only $y / h<0.1$ has been included in the experimental data to avoid contamination by points in the outer layer.
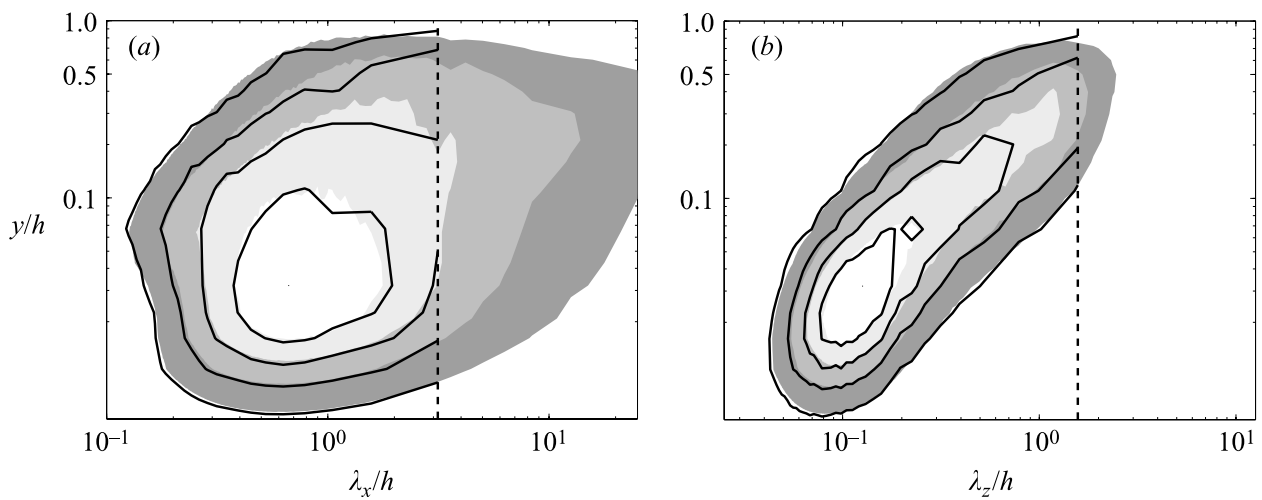

Figure 2. Premultiplied one-dimensional $u v$-cospectra $k\left|E_{u v}^{1 D}\right|^{+}$as functions of wavelength $\lambda$ and wall distance. Shaded contours, case L950; line contours, case S950. The contours are $0.2(0.2) 0.8$ times the maximum value from case L950. The dashed lines mark the size of the smaller box.

of the Reynolds stress $\langle u v\rangle$ which are contained in the simulations L550 and L950 at scales larger than the size of the smaller boxes (i.e. in wavelengths either longer than $\lambda_{x}=\pi h$ or wider than $\lambda_{z}=\pi h / 2$ ), are respectively $51 \%$ and $47 \%$ when averaged across the whole channel.

This result agrees with recent evidence that there are important large-scale contributions to $\langle u v\rangle$ (Jiménez 1998; del Álamo \& Jiménez 2001; Liu, Adrian \& Hanratty 2001), and is probably the reason why the mean-velocity defects from cases S550, S950 and S1900 do not scale as accurately as those from cases L550 and L950 (figure 1a). However, figure 2 suggests that the large-scale contributions to $\langle u v\rangle$ take place mostly in the outer region, in agreement with Townsend's (1976) idea that the impermeability of the wall limits the large-scale motions with a wall-normal component. This figure displays the premultiplied one-dimensional $u v$-cospectra, $k\left\langle\hat{u}(k, y) \hat{v}^{*}(k, y)\right\rangle$, where $\hat{u}$ and $\hat{v}$ are the Fourier coefficients of $u$ and $v$ and $k=2 \pi / \lambda$ are either streamwise or 

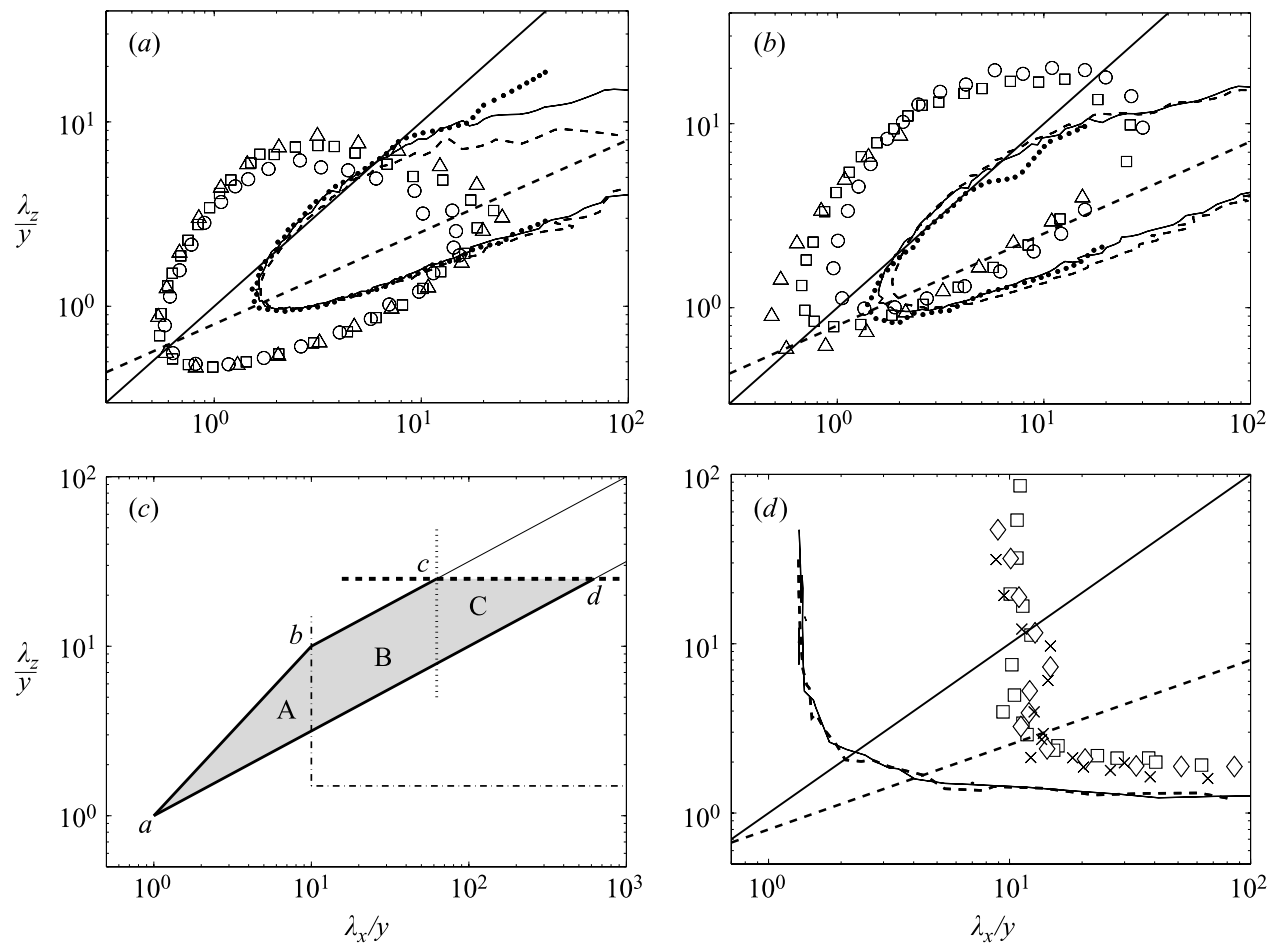

Figure 3. $(a, b)$ Two-dimensional spectral densities $\Phi^{+}$as functions of $\lambda_{x} / y$ and $\lambda_{z} / y$. The line contours are $\Phi_{u u}^{+}=0.17$. (a) $y^{+}=150$ and the symbols are $\Phi_{v v}^{+}=0.06,(b) y / h=0.15$ and the symbols are $\Phi_{w w}^{+}=0.08$. These levels are roughly $5 \%$ of the corresponding intensities. ,--- O, case L550; $\square, \square$, L950; ......., $\triangle$, S1900. The solid straight line is $\lambda_{x}=\lambda_{z}$, and the dashed one is $\lambda_{z}^{2}=2 \kappa \lambda_{x} y$. (c) Sketch of the organization of $\Phi_{u u}$, as discussed in text. (d) Isocontours of correlation coefficient $C_{v v}=1 / 3$ from case L950. Symbols, $C_{v v}\left(15^{+}, y\right)$ : $\square$, $y^{+}=100 ; \diamond, y^{+}=200 ; \times, y^{+}=300$. Lines, $C_{v v}(y / 2, y):-, y^{+}=200 ;---, y^{+}=300$. The correlation increases from left to right, and the straight lines are as in $(a)$.

spanwise wavenumbers. Figure 2 also shows that the misrepresentation of the large scales in the outer layer of the channel for case S950 does not affect substantially the resolved part of the $u v$-cospectrum, especially as we move toward the wall. This is also true for $R e_{\tau}=550$, and it seems reasonable to expect the same to hold for $R e_{\tau}=1900$, particularly for the $O(y)$ scales of the overlap region. This assumption is confirmed by figure $1(b)$, which displays the deviations of the mean-velocity profiles from a logarithmic law $\Psi=U^{+}-\kappa^{-1} \log \left(y^{+}\right)$, using $\kappa=0.4$. It suggests that the overlap region of case S1900 has approximately reached the typical behaviour of high-Reynolds-number experimental wall flows, which are represented in the figure by the hatched and shaded regions. That conclusion, which will be further confirmed throughout the paper, is not very sensitive to the precise value chosen for $\kappa$.

\section{Results and discussion}

Figures $3(a)$ and $3(b)$ show isolines of the premultiplied two-dimensional spectrum of the streamwise velocity, $\Phi_{u u}\left(k_{x}, k_{z}, y\right)=k_{x} k_{z}\left\langle\hat{u} \hat{u}^{*}\right\rangle$, as well as of $\Phi_{v v}$ and $\Phi_{w w}$. Because of the range of $R e_{\tau}$ in the numerical channels, figure 3(a) spans a factor of more than 3 in $y / h$, while figure 3(b) does the same for $y^{+}$. Both scale well with $u_{\tau}^{2}$ and $y$ in the range $y<\lambda_{x}<10 y$. 
However, the ridge of $\Phi_{u u}$ lies along $\lambda_{z} \sim\left(y \lambda_{x}\right)^{1 / 2}$, and needs some discussion because self-similarity would seem to require that $\lambda_{z} \sim \lambda_{x}$. A similar square-root behaviour was found for the width of the near-wall streaks by Jiménez et al. (2003), who showed that its most probable cause was the spreading, under the effect of lateral fluctuations, of wakes formed by compact $v$-structures. Although the outer flow is less coherent than the near-wall layer, and the details of the spreading are probably somewhat different, the source of the square-root behaviour is in both cases the long-term dispersion by background turbulence (Townsend 1976, p. 337).

Consider the random stirring of the mean velocity gradient by active eddies of size $O(y)$ and intensity $O\left(u_{\tau}\right)$, which are represented by the spectral densities $\Phi_{v v}$ and $\Phi_{w w}$ in figures $3(a)$ and $3(b)$. The expected lateral deviations of the fluid elements caused by those eddies at long times is $\lambda_{z} \approx\left(2 v_{T} t\right)^{1 / 2}$, where $v_{T} \approx \kappa u_{\tau} y$ is the turbulent eddy diffusivity in the logarithmic region. As the wakes lengthen, time is converted into wavelength as $\lambda_{x}=\Delta U t$, where $\Delta U$ is the difference between the mean flow and the advection velocity of the forcing fluctuations. There are few data on the advection velocities of $v$ and $w$ in the logarithmic layer, but Kim \& Hussain (1993) find that they differ from the local mean velocity by at most a few wall units (see also del Álamo et al. 2004). This leads to the desired relation, $\lambda_{z}^{2}=2 \kappa \lambda_{x} y / \Delta U^{+}$, which is represented in figures $3(a)$ and $3(b)$ with $\Delta U^{+}=1$. As the wakes widen they also grow in height, although the $y$-dependence of $v_{T}$ leads then to a linear growth.

Refer now to the diagram in figure 3(c). Besides the two square-root segments $b c$ and $a d, \Phi_{u u}$ is bounded at short wavelengths by the segment $a b$ along $\lambda_{z} \approx \lambda_{x}$, which represents the linear dispersion of fluid elements for separations shorter than the integral scale of the active eddies (see below). This scale is of the order of $\lambda_{x}=10 y$, and marks both the transition between the linear and square-root behaviours of $\Phi_{u u}$, and the long-wave cut-offs of $\Phi_{v v}$ and $\Phi_{w w}$. It is also the border between wall-attached and wall-detached eddies. Consider the isocontours in figure $3(d)$ of the correlation coefficient of $\hat{v}$ at two heights, defined as

$$
C_{v v}=\left|\left\langle\hat{v}\left(\lambda_{x}, \lambda_{z}, y_{0}\right) \hat{v}^{*}\left(\lambda_{x}, \lambda_{z}, y\right)\right\rangle\right| /\left(\left\langle\left|\hat{v}\left(\lambda_{x}, \lambda_{z}, y_{0}\right)\right|^{2}\right\rangle\left\langle\left|\hat{v}\left(\lambda_{x}, \lambda_{z}, y\right)\right|^{2}\right\rangle\right)^{1 / 2} .
$$

The lines in figure $3(d)$ indicate that when $y_{0} \sim y\left(y_{0}=y / 2\right.$ for the lines shown), and both $y$ and $y_{0}$ are in the logarithmic layer, the $\lambda$ dependence of the correlations scales with $y$. This breaks down however if $\lambda$ is much larger than $h$. Similarly, with $y_{0}$ fixed in the viscous layer $\left(y_{0}^{+}=15\right.$ for the symbols in figure $\left.3 d\right)$, the wavelength dependence also scales with $y$, but the region of significant correlation is shifted to much larger $\lambda_{x}$. This result, which was also observed for $C_{u u}$ and $C_{w w}$, suggests that there is a range of wavelengths which are correlated within the overlap layer, but not with the wall, and which are therefore 'detached' in the sense of Townsend (1976). The detached wavelength range extends between the two sets of contours in figure $3(d)$, and is bounded for large wavelengths at $\lambda_{x} \approx 10 y$, because for such wavelengths, correlations reach to the wall. It also contains all the energetic scales for $v$ as shown in figure $3(a)$. This is consistent with the idea that blocking by the wall limits the size of the active wall-normal motions, and explains the transition from linear to square-root behaviour of $\Phi_{u u}$. At long wavelengths the $u$-spectrum is no longer forced by the active eddies, and only the incoherent square-root behaviour remains. The ridge of $\Phi_{u u}$, which does not contain $\Phi_{v v}$ nor $\Phi_{w w}$, corresponds to Townsend's (1976) attached inactive motions. The limit that separates active and inactive eddies is represented by the chain-dotted lines in figure $3(c)$. 

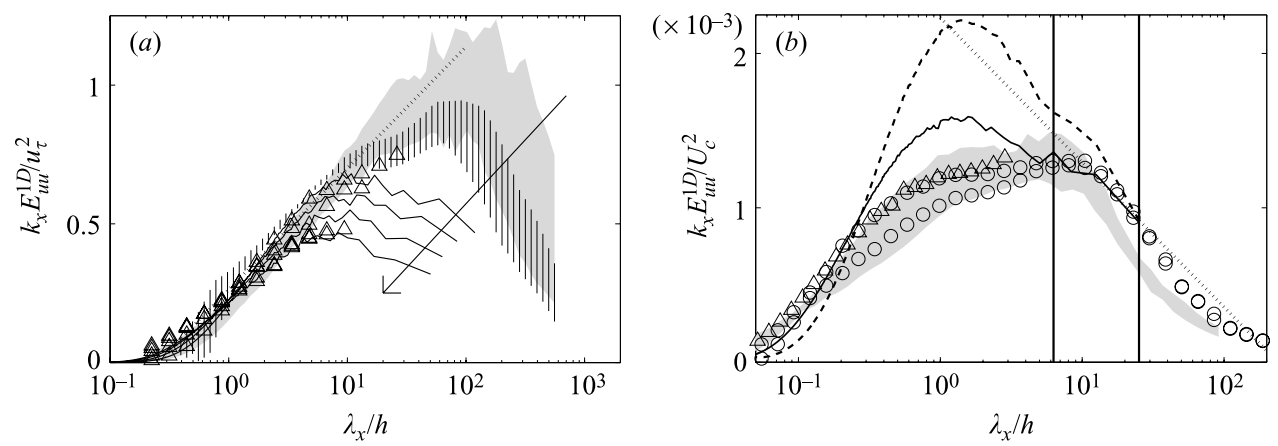

FIGURE 4. Premultiplied one-dimensional spectra $k_{x} E_{u u}^{1 D}$ as functions of the streamwise wavelength $\lambda_{x}$. (a) Scaled with similarity variables $u_{\tau}$ and $y$. The distance to the wall, $y^{+}>200$, increases in the sense of the arrow. Only $y / h<0.1$ has been considered in the experiments to avoid contamination by the points in the outer layer, while up to $y / h=0.5$ has been represented for the DNS. The dotted straight line is $0.2 \log \left(4 \lambda_{x} / y\right)$. (b) Scaled with outer variables $U_{c}$ and $h, y / h=0.1$. The dotted straight line is $5 \times 10^{-4} \log \left[24 h^{2} /\left(\lambda_{x} y\right)\right]$. The solid vertical lines mark the band $6<\lambda_{x} / h<24$ used to obtain figure 5 and (3.3). ------, L550; - L950; $\triangle$, S1900. The shaded areas cover the maximum scatter of experimental boundary layers from Hites (1997), $R e_{\tau}=1300-7100$. The circles and the hatched regions are for pipe flow from Perry \& Abell (1975). $R e_{\tau}=2325-4900$.

Note that, because $\langle u v\rangle$ is mostly generated by the detached eddies in the range A of the spectrum shown in figure $3(c)$, the intensity of the fluctuations in that region should scale well with $u_{\tau}$. This is consistent with the collapse of the different Reynolds numbers in figures $3(a)$ and $3(b)$, and also holds for other spectral and correlation isolines.

The only limit in figure 3(c) which does not scale with $y$ is the dashed horizontal line $\lambda_{z}=\lambda_{z c}$, that fixes the maximum width of $\Phi_{u u}$. Del Álamo \& Jiménez (2003) found that in channels $\lambda_{z c} \approx 2 h$ to $3 h$, which is probably due to the saturation in the growth of the $u$-eddies as they reach the centre of the channel.

A consequence of these scalings is a logarithmic correction to the $k_{x}^{-1}$ onedimensional $u$-spectrum in the range of the detached eddies. Integrating $\Phi_{u u}$ over $\lambda_{z}$ we obtain,

$$
k_{x} E_{u u}^{1 D} \approx \int_{\lambda_{a d}}^{\lambda_{a b}} \Phi_{u u} \frac{\mathrm{d} \lambda_{z}}{\lambda_{z}} \approx \beta u_{\tau}^{2} \log \left(\alpha^{2} \lambda_{x} / y\right),
$$

where the two limits are the square-root lower bound $\lambda_{a d} \approx \alpha^{-1}\left(\lambda_{x} y\right)^{1 / 2}$, and the linear upper bound $\lambda_{a b} \approx \lambda_{x}$ in figure 3(c). We have assumed that $\Phi_{u u} \approx u_{\tau}^{2}$ is roughly constant inside those limits, which is a reasonable approximation to the data. The logarithmic correction reflects the different scalings of the two limits, and figure 4(a) shows that it describes well the numerical and experimental spectra in the range $y<\lambda_{x}<10 y$. The two numerical coefficients $\alpha \approx 2$ and $\beta \approx 0.2$ in (3.2) have been chosen to fit the data in figure $4(a)$, but the resulting value for $\alpha$ is also consistent with the position of the lower limit of $\Phi_{u u}$ in figures $3(a)$ and $3(b)$.

The other spectral range in which the scaling is reasonably clear is region $\mathrm{C}$ in figure 3(c), which corresponds to the 'global' modes described by Bullock, Cooper \& Abernathy (1978) and by del Álamo \& Jiménez (2003) as being correlated across the entire flow. There is no reason why these modes, which carry little Reynolds stress, should scale with $u_{\tau}$ and, because their large correlation heights suggest that they originate from the stirring of the whole velocity profile, from the wall to the top 

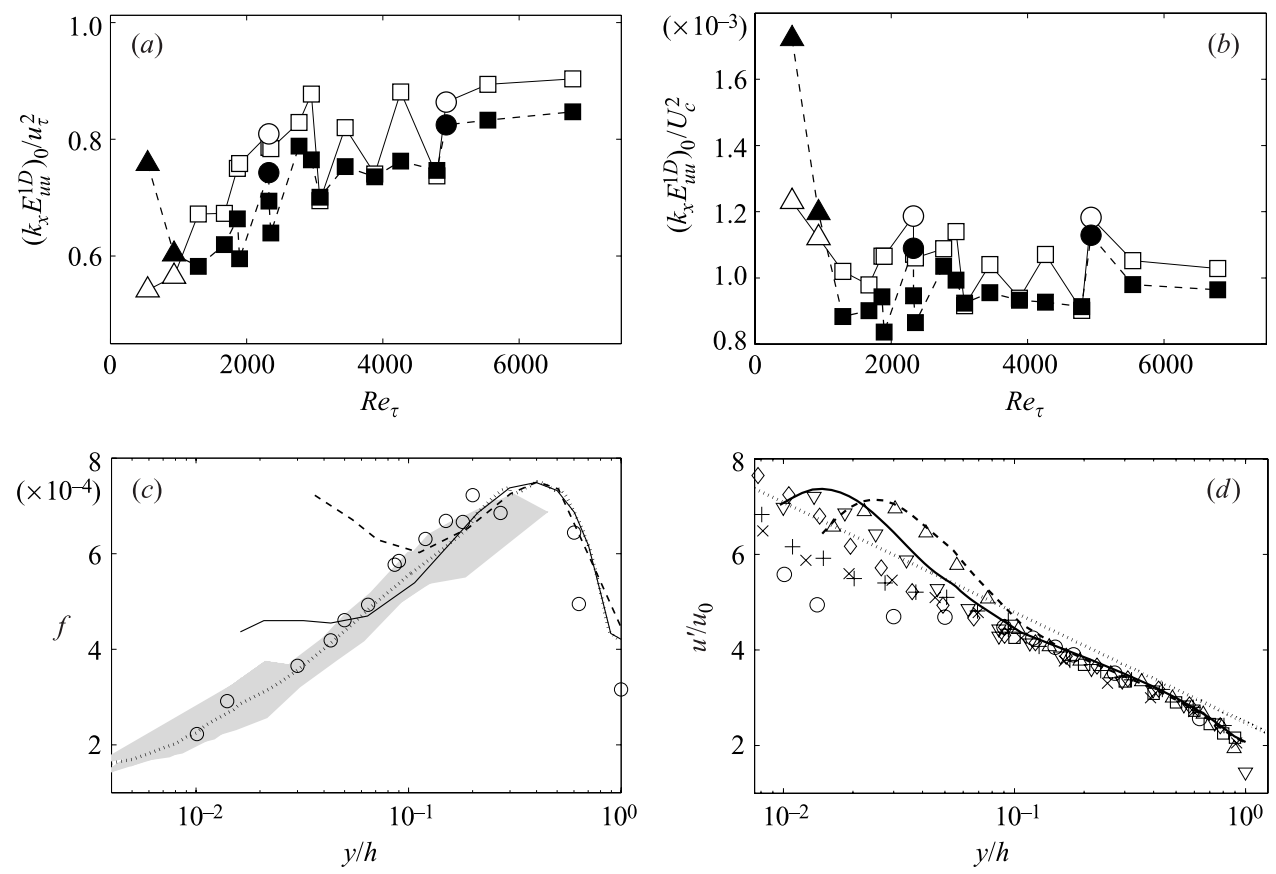

FiguRE 5. $(a, b)$ Average value, $\left(k_{x} E_{u u}^{1 D}\right)_{0}$, of the premultiplied one-dimensional spectrum in the band $6<\lambda_{x} / h<24$ as a function of $R e_{\tau}$. Solid symbols, $y / h=0.05$; open symbols, $y / h=0.1$. $\triangle$, L550 and L950; O, Perry \& Abell (1975); $\square$, Hites (1997). (a) Scaled with $u_{\tau}$, (b) scaled with $U_{c}$. $(c)$ Amplitude $f(y / h)$ of the global modes in (3.3). Shaded areas and circles are as in figure $4 ; \cdots \cdots \cdots$, the composite function used in $(d)$ for $u_{0}^{2} .(d)$ R.m.s of the streamwise velocity $u^{\prime}$ scaled with $u_{0}$ from (3.5) as a function of wall-distance $y / h . \cdots \cdots, \log (12 h / y)$. Boundary layers from de Graaff \& Eaton (2000): $\triangle, R e_{\tau}=540 ; \nabla, R e_{\tau}=990 ; \diamond, R e_{\tau}=1700$; ,$+ R e_{\tau}=4300 ; \times, R e_{\tau}=10^{4} ; \bigcirc$, pipe flow from Perry \& Abell (1975), $R e_{\tau}=4930 ; \square$, channel flow from Comte-Bellot (1965), $R e_{\tau}=8160$. In $(c)$ and $(d)---$, L550; — , L950. Only $y^{+}>15$ has been considered.

of the flow, a more plausible scale is the maximum mean velocity $U_{c}$. Figure $4(b)$ shows that $h$ and $U_{c}^{2}$ are indeed reasonable scales for the one-dimensional spectra when $\lambda_{x} / h>10$. There is some variation between internal and external flows, to be expected in these global modes, and even the long numerical boxes of cases L550 and L950 do not extend to the longest scales, but the collapse is convincing in the common region marked in the figure by the two vertical lines. This is shown in figures $5(a)$ and $5(b)$, which display the average energy density of $u$ in that spectral band as a function of Reynolds number. This magnitude is represented at two wall distances which include the one in figure $4(b)$. When it is scaled with $u_{\tau}^{2}$ it increases with $R e_{\tau}$ by approximately a factor of 2 , but it remains roughly constant when scaled with $U_{c}^{2}$, except for the lowest Reynolds number.

Computing the one-dimensional spectrum in the range $\mathrm{C}$ in figure $3(\mathrm{c})$, in the same way as was done in (3.2), we obtain

$$
k_{x} E_{u u}^{1 D} \approx \int_{\lambda_{a d}}^{\lambda_{z c}} \Phi_{u u} \frac{\mathrm{d} \lambda_{z}}{\lambda_{z}} \approx U_{c}^{2} f(y / h) \log \left(\frac{\alpha^{2} \lambda_{z c}^{2}}{\lambda_{x} y}\right),
$$

which includes a logarithm for the same reason as before, i.e. that the two limits scale with a different power of $\lambda_{x}$. Equation (3.3) is included in figure $4(b)$ as the dotted straight line, and represents the data reasonably well but, in contrast to the 
empirical amplitude $\beta$ in the short-wave range in (3.2), the amplitude $f(y / h)$ in (3.3) depends on $y / h$. This is because, while the short detached structures are self-similar in the sense of being unaffected by boundary conditions, those in region $\mathrm{C}$ represent different levels in global eddies spanning the whole flow height, and have a definite vertical structure. The function $f(y / h)$ has been computed in figure $5(c)$ for various flows by comparing the energy contained for each of them in the spectral band $6 h<\lambda_{x}<24 h$ with model (3.3), and is fairly universal. It is shown by del Álamo et al. (2004) that the vertical structure of all the attached modes in $\mathrm{C}$ is essentially the same. The function $f(y / h)$, as used in (3.3), is a representation of that structure.

The factor $y$ in the denominator inside the logarithm of (3.3) is due to the displacement with wall distance of the upper bound $c d$ of region $\mathrm{C}$ in figure $3(c)$, which progressively 'cuts' $\Phi_{u и}$ at lower values of $\lambda / y$. This effect, combined with the variation of $f$, produces the shortening of the peak of the one-dimensional spectrum as $y$ increases through the outer layer, which has been documented by various groups, probably first by Perry \& Abell (1975).

One result of this 'cutting' effect is that above $y>\lambda_{z c} / 10 \approx 0.2 h-0.3 h$, region B of the spectrum disappears, and A touches C. This part of the flow is particularly easy to analyse, and the total energy of the fluctuations can be obtained by integrating (3.2) for $\lambda_{x}<\lambda_{z c}$ and (3.3) for $\lambda_{x}>\lambda_{z c}$. This results in

$$
u^{\prime 2} \approx u_{0}^{2} \log ^{2}\left(\alpha^{2} \lambda_{z c} / y\right),
$$

where $u_{0}$ is a mixed velocity scale

$$
u_{0}^{2}=\left[\beta u_{\tau}^{2}+f(y / h) U_{c}^{+2}\right] / 2,
$$

which depends on the wall distance, and on the Reynolds number through $U_{c}^{+}$. Figure $5(d)$ applies this scaling to the present simulations and to the measurements of de Graaff \& Eaton (2000), Perry \& Abell (1975) and Comte-Bellot (1965) in boundary layers, pipes and channels. The value of $f(y / h)$ that has been used is the composite one represented in figure $5(c)$ by the dotted curve. The data in figure $5(d)$ collapse reasonably well to (3.4) in the outer layer, even though Wosnik, Castillo $\&$ George (2000) have shown that other flow features differ in that region between boundary layers and parallel flows. Because of the slow growth of $U_{c}$ with $R e_{\tau}$, the velocity scale (3.5) roughly coincides, in the range of Reynolds numbers considered in figure $5(d)$, with the one proposed by de Graaff \& Eaton $(2000), u^{\prime+2} \sim U_{c}^{+}$, but it tends to $u_{0}^{+2}=f U_{c}^{+^{2}} / 2$ when $U_{c}^{+} \gtrsim 50$, roughly $R e_{\tau} \gtrsim 10^{8}$. For such high Reynolds numbers, the one-dimensional spectrum would show a 'discontinuity' between (3.2) and (3.3) around $\lambda_{x} \approx \lambda_{z c}$, marking the wavelength beyond which the structures cross the relatively sharp velocity drop near the wall. There are no reliable measurements of $u^{\prime}$ in the outer region at these Reynolds numbers, but the 'nose' visible in the higher spectra in figure 4(a) could conceivably be an indication of such a mismatch.

Metzger et al. (2001) found that $u^{\prime+^{2}}$ near the wall still scales as $U_{c}^{+}$at Reynolds numbers of order $10^{8}$, but the model that led to (3.5) does not apply in the buffer layer, leading to different conclusions on the scaling. The structure of $\Phi_{u u}$ in that region is described by Jiménez et al. (2003), and is more complex than in figure 3(c). It has a local component which scales in viscous units and which is approximately independent of the outer flow, superimposed on the square-root and global parts B and $\mathrm{C}$. The interactions between those three components are not clear, but there are two effects which do not have a counterpart in the outer region. First, that $f(y / h)$ vanishes at the wall, so that the global modes at a given $y^{+}$do not necessarily increase 
with Reynolds number. Second, that the scale separation between the streaks and the other two flow components grows with increasing Reynolds number.

Another region in which no simple scaling appears to be possible is region $\mathrm{B}$ in figure $3(c)$, which is approximately the range where the classical $k_{x}^{-1}$ spectrum was first documented. Perry, Henbest \& Chong (1986) mentioned what they considered small deviations to the self-similar spectrum at low wavenumbers, and Morrison et al. (2002) proposed that one of the reasons for those deviations could be that the velocity scales of the inner and the outer motions are different. The present results are consistent with that interpretation. The same argument used for the global modes implies that the velocity scale for an attached eddy of height $y_{M}$ should not be $u_{\tau}$, but the mean velocity $U\left(y_{M}\right)$. It is then difficult to define a single velocity scale for the spectrum in region $\mathrm{B}$, which contains contributions from attached eddies of various heights. Only below $\lambda_{x} \approx 10 y$, where the eddies detach from the wall, and above $\lambda_{x} \approx h$, where only eddies of height $h$ exist, do we recover a single scaling. In between the two ranges we can only expect a complex transition between the two regimes, which the limited data at our disposal prevent us from exploring. The data in figures $4(a)$ and 4(b), and those presented by Hites (1997) and Morrison et al. (2002) are consistent with this interpretation, since the latter in particular should have shown a $k_{x}^{-1}$ range if there were one.

\section{Conclusions}

By using new results from direct simulations of turbulent channels at higher Reynolds numbers and in larger boxes than those available up to now, we have probed the corrections to the similarity assumptions in the logarithmic and outer layers of wall-bounded turbulence.

It is shown that the first deviation from simple self-similarity occurs in the width of the $u$-structures, which scales as the square root of their length because $u$ acts like a passive quantity dispersed by the background active turbulence. This anomalous scaling results in logarithmic corrections to various ranges of the $k^{-1}$ spectrum, which have been used to collapse the numerical data with experiments at higher Reynolds numbers.

A second non-classical scaling is the characteristic velocity of the largest $u$ structures, which are global enough to scale with the velocity of the mean flow, instead of with the friction velocity. This is again confirmed by experiments.

The resulting spectral model can be integrated to obtain a mixed scaling for the total intensity of the fluctuating velocity, which collapses the numerical results well with those of de Graaff \& Eaton (2000) in the outer layer, and which predicts that this part of the flow will tend to scale with the mean-stream velocity at very high Reynolds numbers. These results do not extend to the near-wall region. The model also explains other features of the energy spectrum, and is a manifestation of the outer layers themselves, essentially unrelated to their interaction with the near-wall region. As such, it should also essentially apply to other cases, such as flows over rough or manipulated walls, in which the dynamics of the near-wall layer are different from the present case. According to this argument, the only influence of the wall region on the dynamics of the outer layer would come from changes of the velocity scale (3.5).

In Spain this work was supported in part by the CICYT contract BFM2000-1468. The MEC partially supported J.C.A. with a FPU graduate fellowship and R. D.M. with a Sabbatical Program. In the US, this work was supported by the AFOSR under 
grant F49620-01-1-0181, the NFS under grant CTS-001435, and by the Centre for Simulation of Advanced Rockets, which is funded by the DOE through University of California grant B341494. We are especially indebted to the CEPBA/IBM centre of the U. Politècnica de Catalunya, to the San Diego Supercomputer Centre and the US Department of Defence Major Shared Resource Centres, who provided computer time for the remaining ones.

\section{REFERENCES}

Del Álamo, J. C. \& JimÉnEZ, J. 2001 Direct numerical simulation of the very large anisotropic scales in a turbulent channel. In CTR Annual Research Briefs, pp. 329-341. Stanford University.

Del Álamo, J. C. \& JimÉnez, J. 2003 Spectra of the very large anisotropic scales in turbulent channels. Phys. Fluids 15, L41-L44.

del Álamo, J. C., Jiménez, J., Zandonade, P. \& Moser, R. D. 2004 The organization of the logarithmic and outer regions of turbulent channels. In preparation.

Bullock, K. J., CoOper, R. E. \& Abernathy, F. H. 1978 Structural similarity in radial correlations and spectra of longitudinal velocity fluctuations in pipe flow. J. Fluid Mech. 88, 585-608.

Comte-Bellot, G. 1965 Écoulement Turbulent entre Deux Parois Parellèles. Publications Scientifiques et Techniques 419. Ministère de l'Air.

Durst, F., Jovanovic, J. \& SENDER, J. 1995 LDA measurement in the near-wall region of a turbulent pipe flow. J. Fluid Mech. 295, 305-335.

DE GRAAFF, D. B. \& EATON, J. K. 2000 Reynolds-number scaling of the flat-plate turbulent boundary layer. J. Fluid Mech. 422, 319-346.

Hites, M. H. 1997 Scaling of high-Reynolds turbulent boundary layers in the national diagnostic facility. PhD thesis, Illinois Institute of Technology.

JiMÉNEZ, J. 1998 The largest structures in turbulent wall flows. In CTR Annual Research Briefs, pp. 943-945. Stanford University.

JimÉnez, J., Del Álamo, J. C. \& FloRes, O. 2003 The large-scale dynamics of near-wall turbulence. J. Fluid Mech. In press.

Kim, J. \& Hussain, F. 1993 Propagation velocity of perturbations in turbulent channel flow. Phys. Fluids 6, 695-706.

Kim, J., Moin, P. \& Moser, R. D. 1987 Turbulence statistics in fully developed channel flow at low Reynolds number. J. Fluid Mech. 177, 133-166.

Kim, K. C. \& Adrian, R. 1999 Very large-scale motion in the outer layer. Phys. Fluids 11, 417-422.

LAUFER, J. 1954 The structure of turbulence in fully developed pipe flow. Rep. 1174. NACA.

Liu, Z., Adrian, R. J. \& Hanratty, T. J. 2001 Large-scale modes of turbulent channel flow: transport and structure. J. Fluid Mech. 448, 53-80.

Metzger, M. M., Klewicki, J. C., Bradshaw, K. L. \& Sadr, R. 2001 Scaling the near-wall axial turbulent stress in the zero pressure gradient boundary layer. Phys. Fluids 13, 1819-1821.

Morrison, J. F., Jiang, W., McKeon, B. J. \& Smits, A. J. 2002 Reynolds number dependence of streamwise velocity spectra in turbulent pipe flow. Phys. Rev. Lett. 88, 214501/1-4.

Moser, R. D., KIM, J. \& Mansour, N. N. 1999 Direct numerical simulation of turbulent channel flow up to $\operatorname{Re}_{\tau}=590$. Phys. Fluids 11, 943-945.

Österlund, J. M., Johansson, A. V., Nagib, H. M. \& Hites, M. H. 2000 A note on the overlap region in turbulent boundary layers. Phys. Fluids 12, 1-4.

Perry, A. E. \& Abell, C. J. 1975 Scaling laws for pipe-flow turbulence. J. Fluid Mech. 67, 257-271.

Perry, A. E., Henbest, S. \& Chong, M. S. 1986 A theoretical and experimental study of wall turbulence. J. Fluid Mech. 119, 163-199.

SMITH, R. W. 1994 Effects of Reynolds number on the structure of turbulent boundary layers. PhD thesis, Princeton University.

Townsend, A. A. 1976 The Structure of Turbulent Shear Flows, 2nd Edn. Cambridge University Press.

Wosnik, M., Castillo, L. \& George, W. K. 2000 A theory for turbulent pipe and channel flows. J. Fluid Mech. 421, 115-145.

Zagarola, M. \& Smits, A. J. 1997 Experiments in high Reynolds number turbulent pipe flow. Phys. Rev. Lett. 78, 239. 